\title{
On deformations of cross-sections of a differentiable fibre bundle
}

\author{
By \\ Toshimasa YAGYU \\ (Received Jan. 19, 1963)
}

\section{Introduction}

It is well-known that geometric structures on a topological space can be defined mostly through the notion of $(B, \Gamma)$-structure, where $\Gamma$ is a pseudogroup of local homeomorphisms of a topological space $B$. Particularly for a differentiable manifold, when we take the euclidean space $R^{n}$ as $B$ and some pseudogroup $I_{d}$ of local differentiable transformations of $R^{n}$ as $\Gamma,\left(R^{n}, \Gamma_{d}\right)$-structures are objects of differential geometry. On the other hand, there are also structures defined by cross-sections of differentiable bundles over a differentiable manifold such as Riemannian metric structures. But they are not considered generally as $\left(R^{n}, \Gamma_{d}\right)$-structures. However if we take the space of germs of cross-sections of the product bundle over $R^{n}$ as $B$ and a suitable pseudogroup on it as $\Gamma$, we can regard the structures by cross-sections of the differentiable fibre bundle as $(B, \Gamma)$-structures. ( $\$ 5$.

D. C. Spencer $([10])$ has pointed out without proof that the set of germs of $m$-parameter deformations of a $(B, \Gamma)$-structure may be identified with a 1-cohomology set with coefficients on some sheaf, from the theory of A. Haefliger ([8]). Hence, we can apply this theory to deformations of a cross-section and we have a theorem on deformations of a Riemannian manifold as an example.

We give a direct formulation and proof of Spencer's proposition without such a objectionable condition for our application, that $B$ is paracompact. Though our result (Theorem 3 ) can be 
proved more directly, we treat it from a view point of a general theory of deformations of $(B, \Gamma)$-structures, $(\S \S 1-4)$ and its application. $(\$ \S 5-7)$

\section{$\S 1$. Differentiable $(B, \Gamma)$-structures}

Let $B$ be a topological space with a differentiable structure, i.e. there exists a neighborhood $U$ of each point of $B$ and a homeomorphism $\mathcal{P}_{U}$ from $U$ to an open set of $n$-dimensional euclidean space $R^{n}$ such that $\mathcal{P}_{U} \cdot \mathcal{P}_{V}^{-1}$ is a bidifferentiable transformation on $\mathcal{P}_{V}(U \cap V)$ for $U \cap V \neq \Phi$. ( $B$ is not necessarily separable or paracompact.)

Let $\Gamma$ be some pseudogroup of local bidifferentiable transformations of $B$ and let $M$ be a differentiable manifold. For each open set $U$ of $M$, we set

$$
\begin{aligned}
B(U)=\{\mathcal{P} ; \text { a diffeomorphism, in the sense of differentiable } \\
\text { structures of } M \text { and } B \text {, from } U \text { onto the domain } \\
\text { of an element of } \Gamma\} .
\end{aligned}
$$

We define that $\rho, \psi \in B(U)$ are equivalent if and only if $\rho \cdot \psi^{-1} \in \Gamma$ and we denote the set of the equivalence classes of $B(U)$ by $B / \Gamma(U)$. For $U \supset U^{\prime}$, the restriction induces a correspondence $r_{U^{\prime}}^{U}: B(U) \rightarrow B\left(U^{\prime}\right)$ such that $r_{U^{\prime \prime}}^{U^{\prime}} \cdot r_{U^{\prime}}^{U}=r_{U^{\prime \prime}}^{U}$ for $U \supset U^{\prime} \supset U^{\prime \prime}$ and $\left(r_{U^{\prime}}^{U} \boldsymbol{p}\right)\left(r_{U^{\prime}}^{U}, r\right)^{-1} \in \Gamma$ if y $\cdot \mathcal{P}^{-1} \in \Gamma$. Therefore, there exists a correspondence $r_{U^{\prime}}^{\prime}: B / \Gamma(U)$ $\rightarrow B / \Gamma\left(U^{\prime}\right)$ such that $r_{U^{\prime \prime}}^{\prime \prime} \cdot r_{U^{\prime}}^{\prime}=r_{U^{\prime \prime}}^{\prime \prime}$ for $U \supset U^{\prime} \supset U^{\prime \prime}$. and then $\{B / \Gamma(U)\}$ is a presheaf over $M$ and induces a sheaf $[B / \Gamma]_{M}$ over $M$.

Definition. A differentiable $(B, \Gamma)$-structure on $M$ is an element $s$ of $H^{\circ}\left(M,[B / \Gamma]_{M}\right)$, which is a section of $[B / \Gamma]_{M}$ over $M$.

For a differentiable $(B, \Gamma)$-structure $s$, there exist a suitable open neighborhood $U$ of each point $x$ of $M$ and $s_{U} \in B / \Gamma(U)$ such that the germ of $s_{U}$ at $x$ is $s(x)$, and we have $\rho_{U} \in B(U)$ such that $p_{U}\left(\rho_{U}\right)=s_{U}$ where $p_{U}$ is the projection $B(U) \rightarrow B / \Gamma(U) . \quad U$ and $\mathcal{P}_{U}$ are called a coordinate neighborhood of $s$ and coordinate map of $s$, respectively. For an open covering $\left\{U_{j}, j \in J\right\}$ of $M$ by coordinate neighborhoods of $s$ and coordinate maps $\varphi_{j} \in B\left(U_{j}\right),\left\{U_{j}, \varphi_{j}, j \in J\right\}$ is called a coordinate system of $s$. This definition ensures that 
each element of $H^{0}\left(M,[B / \Gamma]_{M}\right)$ has necessarily a coordinate system. If $\left\{U_{k}^{\prime}, k \in K\right\}$ is a refinement of $\left\{U_{i}, j \in J\right\}$ (with the index injection of the refinement $\kappa ; K \rightarrow J)$, then $\left\{U_{k}^{\prime}, \Phi_{k(k)} \mid U_{k}^{\prime}\right\}$ is also a coordinate system of $s$. If $\left\{U_{j}, \mathscr{P}_{j}, j \in J\right\}$ and $\left\{U_{k}^{\prime}, \mathscr{P}_{k}^{\prime}, k \in K\right\}$ are coordinate systems of the same element of $H^{0}\left(M,[B / \Gamma]_{M}\right)$, there exists a refinement $\left\{U_{l}^{\prime \prime}, l \in L\right\}$ of $\left\{U_{j}\right\}$ and $\left\{U_{k}^{\prime}\right\}$ (with the index injections of the refinement $\iota: L \rightarrow J, \kappa: L \rightarrow K)$ such that $\mathcal{P}_{\iota(l)} \mid U_{l}^{\prime \prime}$ and $\mathcal{P}_{\kappa}^{\prime}(l) \mid U_{\imath}^{\prime \prime}$ are equivalent in $B\left(U_{l}^{\prime \prime}\right)$.

Lemma 1. Let $B^{\prime}(U)$ be a subset of $B(U)$ for each open set $U$ of $M$ such that $r_{U^{\prime}}^{U}\left(B^{\prime}(U)\right) \subset B^{\prime}\left(U^{\prime}\right)$ if $U \supset U^{\prime}$, and let $\Gamma^{\prime}$ be a subpseudogroup of $\Gamma$ such that $\rho \cdot \psi \mu^{-1} \in \Gamma^{\prime}$ if $\varphi, \psi \in B^{\prime}(U)$ and $\varphi \cdot \psi^{-1} \in \Gamma$. Then $\left[B^{\prime} / \Gamma^{\prime}\right]_{M}$ is a sub-sheaf of $[B / \Gamma]_{M}$ and so $H^{0}\left(M,\left[B^{\prime} / \Gamma^{\prime}\right]_{M}\right)$ can be identified with a subset of $\left.H^{\circ}(M,[B / \Gamma])_{M}\right)$.

Proof. If $\mathcal{P}, \psi \in B^{\prime}(U)$ are equivalent in $B(U)$, they are equivalent in $B^{\prime}(U)$, and then $B^{\prime} / \Gamma^{\prime}(U) \subset B / \Gamma(U)$. Since $r_{U^{\prime}}^{U}\left(B^{\prime}(U)\right) \subset$ $B^{\prime}\left(U^{\prime}\right), r_{U^{\prime}}^{\prime}: B / \Gamma(U) \rightarrow B / \Gamma\left(U^{\prime}\right)$ maps $B^{\prime} / \Gamma^{\prime}(U)$ into $B^{\prime} / \Gamma^{\prime}\left(U^{\prime}\right)$. Therefore, $\left\{B^{\prime} / \Gamma^{\prime}(U)\right\}$ is a sub-presheaf of $\{B / \Gamma(U)\}$ and so $\left[B^{\prime} / \Gamma^{\prime}\right]_{M}$ is a sub-sheaf of $[B / \Gamma]_{M}$.

When $W$ is an open set of $M$, we define similarly a coordinate system of a section $s \mid W$ of $[B / \Gamma]_{M}$ over $W$.

Lemma 2. Let $\eta$ be a diffeomorphism of $W$ onto an open set of $M$. Then $\eta$ induces a map $\bar{\eta}$ of sections over $\eta(W)$ into sections over $W$.

Proof. If $\left\{U_{j}, \mathscr{P}_{j}\right\}$ is a coordinate system of a section $s \mid \eta(W)$ over $\eta(W), \varphi_{j} \cdot \eta: \eta^{-1}\left(U_{j}\right) \rightarrow B$ is an element of $B\left(\eta^{-1}\left(U_{j}\right)\right)$ and $\left(\mathcal{P}_{i} \eta\right) \cdot\left(\mathcal{P}_{j} \eta\right)^{-1}=\mathcal{P}_{i} \cdot \mathcal{P}_{j}^{-1} \in \Gamma$ for $U_{i} \cap U_{j}(\neq \Phi)$. Therefore $\left\{\mathcal{P}_{j} \cdot \eta, \eta^{-1}\left(U_{j}\right)\right\}$ is a coordinate system of a section over $W$ which is denoted by $\bar{\eta}(s \mid W)$.

Remark. If $\eta$ is a diffeomorphism of $W$ into $M$ such that $\varphi \cdot \eta \in B^{\prime}(U) \subset B(U)$ for any $\rho \in B^{\prime}(\eta(U)) \subset B(\eta(U))$ and any open set $U$ included in $W$, then Lemma 2 ensures that $\eta$ induces a map $\bar{\eta}$ of sections of $\left[B^{\prime} / \Gamma^{\prime}\right]_{M}$ over $\eta(W)$ into sections of $\left[B^{\prime} / \Gamma^{\prime}\right]_{M}$ over $W$. 


\section{§2. Differentiable deformations of $(B, \Gamma)$-structures}

Let $I$ be the open interval $(-1,1)$ of real numbers. The product space $B \times I$ is naturally a topological space with a differentiable structure. Let $\Gamma \times I$ denote the pseudogroup of local bidifferentiable transformations $\gamma$ of $B \times I$ such that

$1^{\circ} . t=\gamma_{t}(x, t)$,

$2^{\circ}$. For every fixed $t$, the local bidifferentiable transformation $\gamma_{x}(x, t)$ of $B$ is an element of the given pseudogroup $\Gamma$ of $B$.

where $\gamma(x, t)=\left(\gamma_{x}(x, t), \gamma_{t}(x, t)\right), x \in B, t \in(-1,1)$.

For each open set $U$ of $M \times I$, we set

$B \times I(U)=\{\mathscr{P}$; diffeomorphisms of $U$ onto domains of elements of $\Gamma \times I$ such that $\varphi_{t}(x, t)$ are independent of $x$ where $\varphi(x, t)=\left(\mathcal{P}_{x}(x, t), \mathcal{P}_{t}(x, t)\right)$ and $\left.(x, t) \in U\right\}$.

$\rho, \psi \in B \times I(U)$ are said to be equivalent if and only if $\rho \cdot \psi^{-1} \in \Gamma \times I$. We set $B \times I / \Gamma \times I(U)=$ \{equivalence classes of $B \times I(U)\}$. Similarly as in $\S 1,\{B \times I / \Gamma \times I(U)\}$ is a presheaf over $M \times I$, and induces a sheaf $[B \times I / \Gamma \times I]_{M, I}$ over $M \times I$.

Let $\left\{U_{j}, \varphi_{j}, j \in J\right\}$ be a coordinate system of $s \in H^{0}(M \times I$, $\left.[B \times I / \Gamma \times I]_{M \times I}\right)$. By the properties of $B \times I(U)$ and $\Gamma \times I, t^{\prime}$ of $\left(y^{\prime}, t^{\prime}\right)=\varphi_{j}(x, 0)$ is a constant for any $j \in J$ and moreover depends only on $s$. We call $t^{\prime}$ the parameter of $s$. We set

$D=\left\{s \in H^{0}\left(M \times I,[B \times I / \Gamma \times I]_{M \times I}\right)\right.$ whose parameter is zero $\}$

Let $\left\{U_{j}, \mathscr{P}_{j}, j \in J\right\}$ denote a coordinate system of an element $s$ of $D$. Setting $V_{j}=U_{j} \cap(M \times 0)$ and identifying $M \times 0$ with $M$, we have $\mathcal{P}_{j} \mid V_{j} \in B\left(V_{j}\right)$ and $\mathscr{P}_{i} \cdot \mathcal{P}_{j}^{-1} \mid \mathcal{P}_{j}\left(V_{i} \cap V_{j}(=\mid=\Phi)\right) \in \Gamma$ since $\mathcal{P}_{j}\left(V_{j}\right) \subset B \times 0$. Therefore $\left\{V_{j}, \mathcal{P}_{j}, j \in J\right\}$ is a coordinate system of an element $s_{0}$ of $H^{0}\left(M,[B / \Gamma]_{M}\right)$ i.e. a differentiable $(B, \Gamma)$-structure. Obviously $s_{0}$ depends only on the element $s$ of $D$ and so we have a map $i: D \rightarrow H^{\circ}\left(M,[B / \Gamma]_{M}\right)$.

Lemma 3. The map $i$ maps $D$ onto $H^{\circ}\left(M,\left[B / \Gamma^{\prime}\right]_{M}\right)$.

Proof. Let $\left\{V_{\alpha}, \psi_{\alpha}, \alpha \in A\right\}$ be a coordinate system of an 
element $s_{0}$ of $H^{\circ}\left(M,[B / \Gamma]_{M}\right)$ and $\varphi_{a}$ denote a map of $V_{a} \times I$ into $B \times I$ defined by

$$
\rho_{\infty}(x, t)=\left(\psi_{\infty}(x), t\right), \quad\left(x \in V_{\alpha}, t \in I\right) .
$$

Hence,

$$
\left(\mathcal{P}_{\alpha} \mid\left(V_{\alpha} \times I\right) \cap\left(V_{\beta} \times I\right)\right)\left(\mathcal{P}_{\beta} \mid\left(V_{\alpha} \times I\right) \cap\left(V_{\beta} \times I\right)\right)^{-1} \in \Gamma \times I
$$

where $V_{\alpha} \cap V_{\beta} \neq \Phi$, and thus $\left\{\varphi_{a}, V_{\alpha} \times I, \alpha \in A\right\}$ is a coordinate system of an element of $H^{\circ}\left(M \times I,[B \times I / \Gamma \times I]_{M \times I}\right)$ and determines $d$ of $D$. Since $\varphi_{a} \mid V_{\alpha}=\psi_{\alpha}$, then $i(d)=s$.

Definition. Differentiable deformations of a given differentiable $(B, \Gamma)$-structure $s_{0}$ are elements $d$ of $D$ such as $i(d)=s_{0}$. We denote their set by $D\left(s_{0}\right)$, i.e. $D\left(s_{0}\right)=i^{-1}\left(s_{0}\right)$.

Let $d_{\varepsilon}$ be a section of $[B \times I / \Gamma \times I]_{M \times I}$ over $M \times(-\varepsilon, \varepsilon)$ where $\varepsilon$ is an arbitrary positive number $(<1)$. $d_{\varepsilon}$ also determines an element of $H^{\circ}\left(M,\left[B / I^{\top}\right]_{M}\right)$.

Lemma 4. When $d_{\mathrm{g}}$ determines an element $s_{0}, d_{\mathrm{g}}$ can be extended to a section of $[B \times I / \Gamma \times I]_{M \times I}$ over $M \times I$ which is an element of $D\left(s_{0}\right)$.

Proof. It is well-known that there exists a diffeomorphism $\eta$ of $M \times I$ on $M \times(-\varepsilon, \varepsilon)$ such that $\eta \mid M \times(-\varepsilon, \varepsilon)=$ identity, $\eta_{x}(x, t)$ is independent of $t$ and $\eta_{t}(x, t)$ is independent of $x$, where $\eta(x, t)=\left(\eta_{x}(x, t), \eta_{t}(x, t)\right)$. If we apply Lemma 2 and Remark of $\S 1$, to $M \times I$ and $\Gamma \times I$, then $\eta$ induces a map $\bar{\eta}$ of sections over $M \times(-\varepsilon, \varepsilon)$ into sections over $M \times 1$, since $\rho \cdot \eta \in B \times I(U)$ for each open set $U$ of $M \times I$ and for $\varphi \in B \times I(\eta(U))$. Then $\bar{\eta}\left(d_{\varepsilon}\right) \in H^{0}(M \times I$, $\left.[B \times I / \Gamma \times I]_{M \times I}\right)$ and moreover $\bar{\eta}\left(d_{\mathfrak{\varepsilon}}\right) \in D\left(s_{0}\right)$ since $\bar{\eta}\left(d_{\mathfrak{\varepsilon}}\right) \mid M \times(-\varepsilon, \varepsilon)$ $=d_{\varepsilon}$.

Henceforth, we suppose that $M$ is compact.

A diffeomorphism $\mathcal{P}$ from an open set $V$ of $M$ to an open set of $B$ is said a regular map on $V$ for a differentiable $(B, \Gamma)$-structure $s_{0}$ if $\left(\mathscr{P}_{j} \mid V_{j} \cap V\right)\left(\mathcal{P} \mid V_{j} \cap V\right)^{-1} \in \Gamma$ for a coordinate system $\left\{V_{j}, \mathscr{\varphi}_{j}\right\}$ of $s_{0}$ and for any $j$ such as $V_{j} \cap V \neq \Phi$. This definition is independent of a coordinate system of $s_{0}$.

For each open set $V$ of $M$ (identified with $M \times 0$ ), we set $\operatorname{II}(V)=\{(\psi, \bar{\gamma})\}$ where $\psi$ is a regular map on $V$ for the given $s_{0}$ 
and $\bar{\gamma}$ is the germ of $\gamma \in \Gamma \times I$ on $\psi(V)$ where the domain of $\gamma$ includes $\psi(V)$. For $\left(\psi^{1}, \bar{\gamma}^{1}\right), \quad\left(\psi^{2}, \bar{\gamma}^{2}\right) \in \Pi(V)$, let the product $\left(\psi^{2}, \bar{\gamma}^{2}\right) \cdot\left(\psi^{1}, \bar{\gamma}^{1}\right)$ be defined if and only if the regular map $\left(\gamma^{\prime} \mid \psi^{\prime}(V)\right) \cdot \psi^{\prime}$ on $V$ is equal to $\psi^{2}$, in this case $\gamma^{2} \cdot \gamma^{1}$ can be combined in the sense of the pseudogroup $\Gamma \times I$ by a suitable restricution of domain, and we set

$$
\left(\psi^{2}, \bar{\gamma}^{2}\right) \cdot\left(\psi^{1}, \bar{\gamma}^{1}\right)=\left(\psi^{1} \text {, germ of } \gamma^{2} \cdot \gamma^{1} \text { on } \psi^{\prime}(V)\right) \in \Pi(V),
$$

where germs of $\gamma^{i}$ on $\psi^{i}(V)$ is $\bar{\gamma}^{i}(i=1,2)$. By this product $\pi(V)$ is a groupoid. For $V \supset V^{\prime}$, the restriction of $\psi, \bar{\gamma}$ defines a map $\mathrm{II}(V) \rightarrow \mathrm{II}\left(V^{\prime}\right)$ and $\{\mathrm{II}(V)\}$ is a presheaf over $M$ and it induces a sheaf [II] of groupoid over $M$.

For an open covering $\mathfrak{B}=\left\{V_{a}, \alpha \in A\right\}$ of $M$, let $\mathcal{C}^{1}(\mathfrak{B}$, II) denote the set of systems $\left\{\bar{\psi}_{\alpha \beta} \in \Pi\left(V_{\alpha} \cap V_{\beta}\right),\left(V_{\alpha} \cap V_{\beta} \neq \Phi\right)\right\}$ such that

$$
\bar{\psi}_{\alpha \beta} \cdot \bar{\psi}_{\beta \gamma}=\bar{\psi}_{\alpha \gamma} \text { for } V_{\alpha} \cap V_{\beta} \cap V_{\gamma} \neq \Phi \text {. }
$$

$\left\{\bar{\Psi}_{\alpha \beta}\right\},\left\{\bar{\psi}_{\alpha \beta}^{\prime}\right\} \in \mathbb{C}^{1}(\mathfrak{B}, \Pi)$ are said to be cohomologous if there exists $\bar{\psi}_{\alpha} \in \Pi\left(V_{\alpha}\right)$ for each $\alpha$ such as $\bar{\psi}_{\alpha} \cdot \bar{\psi}_{\alpha \beta}=\bar{\psi}_{\alpha \beta}^{\prime} \bar{\psi}_{\beta}$ for $V_{\alpha} \cap V_{\beta}(\neq \Phi)$ and we denote by $\mathfrak{S}^{1}\left(\mathfrak{B}\right.$, II) the set of cohomologous classes of $\mathcal{C}^{1}(\mathfrak{B}$, II). For a refinement $\mathfrak{B}^{\prime}=\left\{V_{\alpha^{\prime}}^{\prime}, \alpha^{\prime} \in A^{\prime}\right\}$ of $\mathfrak{B}$, (with the index injection of the refinement $\mathfrak{a}: A^{\prime} \rightarrow A$ ),

$$
\left\{\bar{\psi}_{a\left(\alpha^{\prime}\right) a\left(\beta^{\prime}\right)} \mid V_{\alpha^{\prime}}^{\prime} \cap V_{\beta^{\prime}}^{\prime}(+\Phi)\right\} \in \mathbb{C}^{1}\left(\mathfrak{B}^{\prime}, \mathrm{II}\right)
$$

and if $\left\{\bar{\psi}_{\alpha, \beta}\right\},\left\{\bar{\psi}_{\alpha, \beta}^{\prime}\right\}$ are cohomologous, then $\left\{\bar{\psi}_{a\left(\alpha^{\prime}\right) a\left(\beta^{\prime}\right)} \mid V_{\alpha^{\prime}}^{\prime} \cap V_{\beta^{\prime}}^{\prime}\right\}$, $\left\{\bar{\psi}_{a\left(\alpha^{\prime}\right) a\left(\beta^{\prime}\right)}^{\prime} \mid V_{a^{\prime}}^{\prime} \cap V_{\beta^{\prime}}^{\prime}\right\}$ are cohomologous in $\mathcal{C}^{1}\left(\mathfrak{B}^{\prime}, 11\right)$. Therefore we have a correspondence

$$
\bar{\gamma}_{\mathfrak{P}^{\prime}}^{\mathfrak{R}}: \mathfrak{S}^{1}(\mathfrak{V}, \mathrm{II}) \rightarrow \mathfrak{S}^{1}\left(\mathfrak{W}^{\prime}, \mathrm{II}\right)
$$

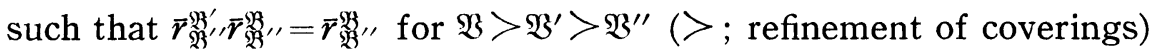
and the system $\left\{\mathfrak{g}^{1}(\mathfrak{B}, \Pi), \mathfrak{B} \in\right.$ the systems of open coverings of $\left.M\right\}$ forms a direct system. We denote its inductive limit by $H^{1}(M,[I I])$. (The Čech cohomology set of 1-dim with coefficients in the sheaf [II]). An element $\left\{\bar{\psi}_{\alpha \beta}\right\}$ of $\mathcal{C}^{1}(\mathfrak{B}, \mathrm{II})$ is called a cocycle of $\bar{\psi}$ for W3 if $\bar{\psi}$ is the element of $H^{1}(M,[11])$ determined by the inductive limit of cohomologous class of $\left\{\bar{\psi}_{\alpha \beta}\right\}$.

Lemma 5. There exists a map $\delta$ from $D\left(s_{0}\right)$ onto $H^{1}(M,[11])$. Proof. If $\left\{U_{j}, \mathscr{P}_{j}, j \in J\right\}$ is a coordinate system of $d \in D\left(s_{0}\right)$, 
then $\rho_{j} \mid V_{j}: V_{j} \rightarrow B \times 0$ (identified with $B$ ) are regular maps for $s_{0}$ where $V_{j}$ denote $U_{j} \cap(M \times 0)(: \mid-\Phi)$, and $\left(\mathscr{P}_{i} \bullet \mathscr{P}_{j}^{-1}\right) \in \Gamma \times I\left(U_{i} \cap U_{j}=\mid \oplus\right)$. Therefore if we denote by $\overline{\mathcal{P}}_{i j}$

$$
\left(\mathcal{P}_{j} \mid V_{j} \text {, germ of } \varphi_{i} \mathcal{P}_{j}^{-1} \text { on } \mathcal{P}_{j}\left(V_{i} \cap V_{j}\right)\right)
$$

then $\quad \overline{\mathcal{P}}_{i j} \in \Pi\left(V_{i} \cap V_{j}\right)$ and $\overline{\mathcal{P}}_{i j} \cdot \overline{\mathcal{P}}_{j k}=\overline{\mathcal{P}}_{i k}$ on $V_{i} \cap V_{j} \cap V_{k} \neq \Phi$. Since $\left\{V_{j}=U_{j} \cap(M \times 0)\right\}$ is a covering of $M,\left\{\overline{\mathcal{P}}_{i j}\right\}$ is a cocycle of an element $\overline{\mathcal{P}}$ of $\left.H^{1}(M,[I]]\right)$. If we take other coordinate system $\left\{U_{k}^{\prime}, \mathcal{P}_{k}^{\prime}, k \in K\right\}$ of $d$, there exists a refinement covering $\left\{U_{\imath}^{\prime \prime}, l \in L\right\}$ of the coverings $\left\{U_{j}, j \in J\right\}$ and $\left\{U_{k}^{\prime}, k \in K\right\}$ (with index injections of refinement $\iota: L \rightarrow J, \kappa: L \rightarrow K)$, then

and

$$
\left(\mathscr{P}_{\imath(l)} \mid U_{\imath}^{\prime \prime}\right)\left(\mathscr{P}_{\kappa(l)}^{\prime} \mid U_{\imath}^{\prime \prime}\right)^{-1} \in I \times I
$$

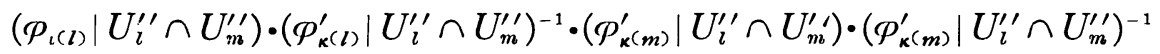
$=\left(\mathcal{P}_{\iota(m)} \mid U_{l}^{\prime \prime} \cap U_{m}^{\prime \prime}\right) \cdot\left(\mathscr{P}_{\iota(m)} \mid U_{l}^{\prime \prime} \cap U_{m}^{\prime \prime}\right)^{-1} \cdot\left(\mathcal{P}_{\iota(m)} \mid U_{l}^{\prime \prime} \cap U_{m}^{\prime \prime}\right) \cdot\left(\mathcal{P}_{\kappa(m)}^{\prime} \mid U_{l}^{\prime \prime} \cap U_{m}^{\prime \prime}\right)^{-1}$ on $U_{l}^{\prime \prime} \cap U_{m}^{\prime \prime} \neq \Phi$. If we set

$$
\bar{\psi}_{l}=\left(\mathscr{P}_{\kappa(l)}^{\prime} \mid V_{l}^{\prime \prime}, \text { germ of } \mathcal{P}_{\iota(l)} \cdot\left(\mathscr{P}_{\kappa(l)}^{\prime}\right)^{-1} \text { on } \mathscr{P}_{\kappa(l)}^{\prime}\left(V_{l}^{\prime \prime}\right)\right)
$$

where $V_{l}^{\prime \prime}=U_{l}^{\prime \prime} \cap(M \times 0)(\neq \Phi)$, then $\bar{\gamma}_{l} \in \Pi\left(V_{l}^{\prime \prime}\right)$ and

$\left(\bar{r}_{l} \mid V_{l}^{\prime \prime} \cap V_{m}^{\prime \prime}\right) \cdot\left(\overline{\mathcal{P}}_{\kappa(l) k(m)}^{\prime} \mid V_{l}^{\prime \prime} \cap V_{m}^{\prime \prime}\right)=\left(\overline{\mathcal{P}}_{\iota(l)_{\iota}(m)} \mid V_{l}^{\prime \prime} \cap V_{m}^{\prime \prime}\right) \cdot\left(\bar{\psi}_{m} \mid V_{l}^{\prime \prime} \cap V_{m}^{\prime \prime}\right)$.

Since $\left\{V_{l}^{\prime \prime}\right\}$ is a refinement of the coverings $\left\{V_{j} \equiv U_{j} \cap(M \times 0)\right\}$ and $\left\{V_{k}^{\prime} \equiv U_{k}^{\prime} \cap(M \times 0)\right\}$ of $M \times 0(\equiv M)$, then $\left\{\overline{\mathcal{P}}_{k n}^{\prime}\right\}$ is a cocycle of the same element $\overline{\mathcal{P}}$ and the correspondence $d \rightarrow \overline{\mathcal{P}}$ defines a map $\delta: D\left(s_{0}\right) \rightarrow H^{1}(M,[\Pi])$.

Next, if $\left\{\bar{\psi}_{\alpha \beta}\right\}=\left\{\left(\psi_{\alpha \beta}, \bar{\gamma}_{\alpha \beta}\right)\right\}$ is a cocycle of an element $\bar{f} \in H^{1}(M,[\Pi])$ for an open finite covering $\left\{V_{\alpha}, \alpha \in A\right\}$ of $M$, there exists a finite covering $\left\{U_{j}, j \in J\right\}$ of $M \times 0$ by open sets of $M \times I$ satisfying following conditions :

1) $\left\{U_{j} \cap(M \times 0)\right\}$ considered as a covering of $M$, is a refineof $\left\{V_{\alpha}\right\}$ (with the index injection of the refinement $a: J \rightarrow A$ )

2) there exist $\mathcal{P}_{i j} \in B \times I\left(U_{i} \cap U_{j}(\neq \Phi)\right)$ and $\gamma_{\alpha \beta} \in \Gamma \times I$ such that (domain of $\left.\gamma_{a(i) a(j)}\right)>\varphi_{i j}\left(U_{i} \cap U_{j}\right), \varphi_{i j}\left(V_{\jmath}^{\prime}\right) \subset B \times 0$ and

$$
\psi_{a(i) a(j)}\left|V_{i}^{\prime} \cap V_{j}^{\prime}=\varphi_{i j}\right| V_{i}^{\prime} \cap V_{j}^{\prime}
$$

where $V_{j}^{\prime}=U_{j} \cap(M \times 0)$ and 


$$
\bar{\gamma}_{a(i) a(j)} \mid \mathcal{P}_{i j}\left(V_{i}^{\prime} \cap V_{j}^{\prime}\right)=\left(\text { germ of } \gamma_{i j} \text { on } \mathscr{P}_{i j}\left(V_{i}^{\prime} \cap V_{j}^{\prime}\right)\right) \text {. }
$$

Since $\bar{\psi}_{\alpha \beta} \bar{\psi}_{\beta \gamma}=\bar{\psi}_{\alpha \gamma}$ on $V_{\alpha} \cap V_{\beta} \cap V_{\gamma}(\vDash \Phi)$ and by the definition of the product in $\Pi(V)$, we can choose these objects such that $\mathcal{P}_{i i} \varphi_{j j}^{-1}=\gamma_{i j} \in \Gamma \times I$ for $U_{i} \cap U_{j} \neq \Phi$ and $\gamma_{i j} \gamma_{j k}=\gamma_{j k}$ for $U_{i} \cap U_{j} \cap U_{k}(\neq=\Phi)$. Since $\left\{U_{j}\right\}$ is a finite covering, we can take a positive number $\varepsilon(<1)$ such that $M \times(-\varepsilon, \varepsilon) \subset \bigcup_{j \in I} U_{j}$. If we set $\mathcal{P}_{j}=\mathscr{P}_{j j} \mid U_{j} \cap$ $(M \times(-\varepsilon, \varepsilon)), \quad\left\{\rho_{j}, U_{j} \cap(M \times(-\varepsilon, \varepsilon), j \in J\}\right.$ is a coordinate system of a section $d_{\mathrm{e}}$ of $[M \times I / \Gamma \times I]_{M \times I}$ over $M \times(-\varepsilon, \varepsilon)$. By Lemma 4 there is a $d \in D\left(s_{0}\right)$ such that $d \mid M \times(-\varepsilon, \varepsilon)=d_{\mathrm{q}}$. Since $\gamma_{i j}=\boldsymbol{P}_{i} \mathcal{P}_{j}^{-1}$ for $\left(U_{i} \cap M \times(-\varepsilon, \varepsilon)\right) \cap\left(U_{j} \cap(M \times(-\varepsilon, \varepsilon)) \neq \Phi\right.$ and so

$$
\mathcal{P}\left\{\left(\mathcal{P}_{i j} \mid V_{i}^{\prime} \cap V_{j}^{\prime} \text {, germ of } \gamma_{i j} \text { on } \mathcal{P}_{i j}\left(V_{i}^{\prime} \cap V_{j}^{\prime}\right)\right)\right\}=\bar{\psi}_{\mathrm{a}(i) a(j)} \mid V_{i}^{\prime} \cap V_{j}^{\prime} \text {, }
$$

then we have $\delta(d)=T$.

\section{$\S 3$. Classes of locally equivalent deformations}

Elements $d$ of $D\left(s_{0}\right)$ being sections of the sheaf $[B \times I / \Gamma \times I]_{M \times I}$, let $d \mid W$ denote their restrictions on an open set $W$ of $M \times I$ and set $D\left(s_{0}\right) \mid W=\left\{d \mid W ; d \in D\left(s_{0}\right)\right\}$. If $\eta$ is a diffeomorphism from an open set $W$ of $M \times I$ into $M \times I$ such that

[1] $\eta_{t}(x, t)$ is independent of $x$

where $\eta(x, t)=\left(\eta_{x}(x, t), \eta_{t}(x, t)\right),((x, t) \in W, x \in M, t \in I)$, then $\eta$ induce a map $\bar{\eta}$ from $D\left(s_{0}\right) \mid \eta(W)$ into $D\left(s_{0}\right) \mid W$.

Definition. Two differentiabl edeformation $d^{1}$ and $d^{2}$ of $s_{0}$ are locally equivalent if there exist a positive number $\varepsilon>1$ and a diffeomophism $\eta$ from $M \times(-\varepsilon, \varepsilon)$ into $M \times I$ such that $\eta$ satisfies [1] and also the following two conditions,

$$
\begin{aligned}
& \eta(x, 0) \text { is identity, } \\
& \bar{\eta}\left(d^{2}\left|\eta(M \times(-\varepsilon, \varepsilon))=d^{1}\right| M \times(-\varepsilon, \varepsilon) .\right.
\end{aligned}
$$

The local equivalence of deformations satisfies the equivalence relation and their equivalence classes are called classes of locally equivalent deformations and the set of these classes is denoted by $\bar{D}\left(s_{0}\right)$.

Proposition 1. The map $\delta: D\left(s_{0}\right) \rightarrow H^{1}(M,[\mathrm{II}])$ induces a bijection $\bar{\delta}: \bar{D}\left(s_{0}\right) \rightarrow H^{1}(M,[\Pi])$. 
Proof. Let $\left\{U_{j}, \varphi_{j}^{1}, j \in J\right\},\left\{U_{j}, \mathscr{P}_{j}^{2}, j \in J\right\}$ denote coordinate systems of deformations $d^{1}, d^{2}$, respectively, for a suitable common covering $\left\{U_{j}, j \in J\right\}$ of $M \times I$. If $d^{1}, d^{2}$ are locally equivalent, there exists a covering $\left\{W_{l}, l \in L\right\}$ of $M \times 0$ (identified with $M$ ) by open sets of $M \times I$, such that

(1) $\left\{W_{l}\right\}$ is a refinement of $\left\{U_{j} ; U_{j} \cap(M \times 0) \neq \Phi\right\}$ as a covering of $M \times 0$ by open sets of $M \times I$ (with the index injection of the refinement $\left.\mu: L \rightarrow J^{\prime} \subset J\right)$,

(2) $W_{l} \subset M \times(-\varepsilon, \varepsilon)$ for each $l \in L$,

(3) $\eta\left(W_{l}\right) \subset U_{\mu(l)}$

where $\eta$ is the diffeomorphism from $M \times(-\varepsilon, \varepsilon)$ into $M \times I$ which gives the local equivalence of $d^{1}, d^{2}$. Since $\left\{\eta\left(W_{l}\right), \mathcal{P}_{(l)}^{2} \mid \eta\left(W_{l}\right)\right\}$ is a coordinate system of $d^{2}\left|\eta\left(\bigcup_{l \in L} W_{l}\right) \subset d^{2}\right| \eta(M \times(-\varepsilon, \varepsilon))$, we see that $\left\{W_{l}, \mathcal{P}_{\mu(l)}^{2} \cdot \eta \mid W_{l}\right\}$ is a coordinate system of $\bar{\eta}\left(d^{2} \mid \eta\left(\bigcup_{l \in L} W_{l}\right)\right)$. On the other hand, $\left\{W_{l}, \rho_{\mu(l)}^{1} \mid W_{l}\right\}$ is a coordinate system of $d^{1} \mid \bigcup_{l \in L} W_{l}$ and $\bar{\eta} \cdot d^{2}\left|\bigcup_{l \in L} W_{l}=d^{1}\right| \bigcup_{l \in L} W_{l}$. Therefore, for each $l \in L$, the local diffeomorphism $\varphi_{\mu(l)}^{2} \cdot \eta \cdot\left(\mathcal{P}_{\mu(l)}^{1}\right)^{-1} \mid \mathcal{P}_{\mu(l)}^{1}\left(W_{l}\right)$ of $B \times I$ is an element of $\Gamma \times I$, and is denoted by $\gamma_{l}$. The image $\varphi_{\mu(l)}^{1}\left(W_{l}\right)$ is the domain of $\gamma_{l}$ and $\mathcal{P}_{\mu(l)}^{1}\left(W_{l}\right) \supset \mathcal{P}_{\mu(l)}^{1}\left(V_{l}\right)$ where $V_{l}=W_{l} \cap(M \times 0)$. Then $\left(\mathcal{P}_{\mu l l}^{1} \mid V_{l}\right.$, germ of $\gamma_{l}$ on $\left.\varphi_{\mu l l}^{1}\left(V_{l}\right)\right) \in \Pi\left(V_{l}\right)$. Since

$$
\begin{gathered}
\mathcal{P}_{\mu(l)}^{1}\left(\mathcal{P}_{\mu(m)}^{1}\right)^{-1} \mid \mathcal{P}_{\mu(m)}^{1}\left(W_{l} \cap W_{m}\right) \in \Gamma \times I, \\
\eta\left(W_{l} \cap W_{m}\right)=\left(\mathcal{P}_{\mu(l)}^{2}\right)^{-1} \cdot \gamma_{l} \cdot \mathcal{P}_{\mu(l)}^{1}\left|W_{l} \cap W_{m}=\left(\mathcal{P}_{\mu(m)}^{2}\right)^{-1} \cdot \gamma_{m} \cdot \mathcal{P}_{\mu(m)}^{1}\right| W_{l} \cap W_{m}
\end{gathered}
$$

and the range of $\gamma_{l}$ is $\varphi_{\mu(l)}^{2}$, then

$$
\gamma_{l} \cdot \mathcal{P}_{\mu(l)}^{1} \cdot\left(\mathcal{P}_{\mu(m)}^{1}\right)^{-1}=\mathcal{P}_{\mu(l)}^{2} \cdot\left(\mathcal{P}_{\mu(m)}^{2}\right)^{-1} \cdot \gamma_{m} \quad \text { on } \quad \mathcal{P}_{\mu(m)}^{1}\left(W_{l} \cap V_{m}\right)
$$

and so $\bar{\psi}_{l} \cdot \overline{\mathcal{P}}_{l m}^{1}, \overline{\mathcal{P}}_{l m}^{2} \cdot \bar{\psi}_{m}$ are defined on $V_{l} \cap V_{m} \neq \Phi$ and are equal, where

$$
\begin{aligned}
& \bar{\psi}_{l}=\left(\mathcal{P}_{\mu(l)}^{1} \mid V_{l}, \text { germ of } \gamma_{l} \text { on } \mathcal{P}_{\mu(l)}^{1}\left(V_{l}\right),\right. \\
& \overline{\mathcal{P}}_{l m}^{1}=\left(\mathcal{P}_{\mu(m)}^{1} \mid V_{m}, \text { germ of } \mathcal{P}_{\mu(l)}^{1} \cdot\left(\mathcal{P}_{\mu(m)}^{1}\right)^{-1} \text { on } \mathcal{P}_{\mu(m)}^{1}\left(V_{m}\right)\right), \\
& \mathcal{P}_{l m}^{2}=\left(\varphi_{\mu(m)}^{2} \cdot \eta \mid V_{m}, \quad \text { germ of } \mathcal{P}_{\mu(l)}^{2}\left(\mathcal{P}_{\mu(m)}^{2}\right)^{-1} \text { on } \mathcal{P}_{\mu(m)}^{2}\left(V_{m}\right)\right) .
\end{aligned}
$$

Therefore, $\left\{\overline{\mathscr{P}}_{l_{m}}^{1}\right\}$ and $\left\{\overline{\mathscr{\rho}}_{l_{m}}^{2}\right\}$ are cohomologous in $\mathcal{C}^{1}\left(\left\{V_{i}\right\}, \Pi\right)$, where the former determines $\delta\left(d^{1}\right)$ and the latter determines $\delta\left(d^{2}\right)$ because $\eta$ is identity on $M \times 0$, that is $\delta\left(d^{1}\right)=\delta\left(d^{2}\right)$. 
Conversely, we suppose $\delta\left(d^{1}\right)=\delta\left(d^{2}\right)$. Since $M$ is compact, there exists a finite covering $\left\{V_{k}, k \in K\right\}=\mathfrak{S}$ of $M$ by open sets of $M$ which is a refinement of $\left\{U_{j} \cap M \times 0(\neq \Phi)\right\}$ as an open covering of $M$ (with the index injection of the refinement $\lambda: K \rightarrow J$ ), such that

$\left\{\mathcal{P}_{k l}^{1}\right\}=\left\{\left(\mathcal{P}_{\lambda(l)}^{1} \mid V_{k} \cap V_{l}(=\mid=\Phi)\right.\right.$, germ of $\mathcal{P}_{\lambda(k)}^{1} \cdot\left(\mathcal{P}_{\lambda(l)}^{1}\right)^{-1}$ on $\left.\left.\mathcal{P}_{\lambda(l)}^{1}\left(V_{k} \cap V_{l}\right)\right)\right\}$ and

$\left\{\overline{\mathcal{P}}_{k l}^{2}\right\}=\left\{\left(\mathcal{P}_{\lambda(l)}^{2} \mid V_{k} \cap V_{l}, \quad\right.\right.$ germ of $\mathcal{P}_{\lambda(k)}^{2} \cdot\left(\mathcal{P}_{\lambda(l)}^{2}\right)^{-1} \quad$ on $\left.\left.\mathcal{P}_{\lambda(l)}^{2}\left(V_{k} \cap V_{l}\right)\right)\right\}$ are cohomologous in $\mathcal{C}^{1}\left(\left\{V_{k}\right\}, \pi\right)$. Then we have a element $\bar{\gamma}_{k}$ of $\Pi\left(V_{k}\right)$ for each $k \in K$ such as

$$
\bar{\gamma}_{k} \cdot \overline{\mathcal{P}}_{k l}^{1}=\overline{\mathcal{P}}_{k l}^{2} \cdot \bar{\gamma}_{l} \quad \text { for } \quad V_{k} \cap V_{l} \neq \Phi .
$$

From the definition of $\pi\left(V_{k}\right)$ and the product in it,

$$
\bar{\gamma}_{k}=\left(\varphi_{\lambda(k)}^{1} \mid V_{k}, \text { germ of } \gamma_{k} \text { on } \varphi_{\lambda(k)}^{1}\left(V_{k}\right)\right)
$$

where $\gamma_{k} \in \Gamma \times I$, (the domain of $\left.\gamma_{k}\right) \cap(B \times 0)=\varphi_{\lambda(k)}^{1}\left(V_{k}\right)$, (the range of $\left.\gamma_{k}\right) \cap(B \times 0)=\varphi_{\lambda(k)}^{2}\left(V_{k}\right)$, and $\gamma_{k} \rho_{\lambda(k)}^{1}\left|V_{k}=\varphi_{\lambda(k)}^{2}\right| V_{k}$. If we set

$W_{k}=\left(\mathcal{P}_{\lambda(k)}^{1}\right)^{-1} \cdot\left(\right.$ the domain of $\left.\gamma_{k}\right) \cap\left(\mathscr{P}_{\lambda(k)}^{2}\right)^{-1} \cdot\left(\right.$ the range of $\left.\gamma_{k}\right)$ $\subset M \times I$,

then $W_{k} \cap(M \times 0)=V_{k},\left\{W_{k}, k \in K\right\}$ is a finite covering of $M \times 0$ by open sets of $M \times I$, and $\left(\mathcal{P}_{\lambda(k)}^{2}\right)^{-1} \cdot \gamma_{k} \cdot \varphi_{\lambda(k)}^{1}$ can be defined on $W_{k}$. Since

then

$$
\bar{\gamma}_{k} \cdot \overline{\mathcal{\rho}}_{\lambda(k) \lambda(l)}^{1}=\overline{\boldsymbol{\rho}}_{\lambda(k) \lambda(l)}^{2} \cdot \bar{\gamma}_{l}
$$

$$
\left(\rho_{\lambda(k)}^{2}\right)^{-1} \cdot \gamma_{k} \cdot \varphi_{\lambda(k)}^{1}=\left(\varphi_{\lambda(k)}^{2}\right)^{-1} \cdot \gamma_{l} \cdot \varphi_{\lambda(l)}^{1} \quad \text { on } \quad W_{k} \cap W_{l}(\neq \Phi) .
$$

Therefore, there exist a positive number $\varepsilon$ and a homeomorphism $\eta$ from $M \times(-\varepsilon, \varepsilon)$ into $M \times I$ such that $M \times(-\varepsilon, \varepsilon) \subset \bigcup_{k \in K} W_{k}$,

$$
\begin{aligned}
& \eta\left|M \times(-\varepsilon, \varepsilon) \cap W_{k}=\left(\mathcal{P}_{\lambda(k)}^{2}\right)^{-1} \cdot \gamma_{k} \cdot \mathcal{P}_{\lambda(k)}^{1}\right|(M \times(-\varepsilon, \varepsilon)) \cap W_{k}, \\
& \gamma_{k} \cdot \mathcal{P}_{\lambda(k)}^{1}\left|M \times(-\varepsilon, \varepsilon) \cap W_{k}=\phi_{\lambda(k)}^{2} \eta\right| M \times(-\varepsilon, \varepsilon) \cap W_{k}
\end{aligned}
$$

and $\eta_{t}(x, t)$ is independent of $x$ where $\eta(x, t)=\left(\eta_{x}(x, t), \eta_{t}(x, t)\right)$. Here, $\left.\left\{\gamma_{k} \cdot \mathcal{P}_{(k)},(M \times(-\varepsilon, \varepsilon)) \cap W_{k}\right)\right\}$ and $\left\{\varphi_{\lambda(k)}^{2} \cdot \eta,\left(M \times(-\varepsilon, \varepsilon) \cap W_{k}\right)\right\}$ are coordinate systems of $d^{1} \mid M \times(-\varepsilon, \varepsilon)$ and $\bar{\eta} d^{2} \mid M \times(-\varepsilon, \varepsilon)$, respectively, i.e. $d^{1} \mid M \times(-\varepsilon, \varepsilon)=\bar{\eta} d^{2}$. Since $\varphi_{\lambda(k)}^{2} \cdot \gamma_{k} \cdot \varphi_{\lambda(k)}^{1}=$ identity 
On deformations of cross-sections of a differentiable fibre bundle 219 on $V_{k}$, then $\eta \mid M \times 0=$ identity. Therefore, $\eta$ gives the local equivalence of $d^{1}$ and $d^{2}$.

\section{\$4. Germs of local automorphisms depending differentiably on 1-parameter for the differentiable $(B, \Gamma)$-structure}

A diffeomorphism $\xi$ of an open set $V$ of $M$ to an open set of $M$ is called a local automorphism for the differentiable $(B, \Gamma)$ structure $s_{0}$ if $\xi \cdot s_{0}=s_{0}$ on $V$, i.e. for a regular map $\varphi$ of $s_{0}$ on a neighborhood of each point $x \in \xi(V), \phi \bullet \xi$ is a regular map on a neighborhood of $\xi^{-1}(x)$.

A diffeomorphism $\zeta$ of $V \times(-\varepsilon, \varepsilon)$ into $M \times(-\varepsilon, \varepsilon)$ is said a local automorphism of $V$ depening differentiably on 1-parameter for $s_{n}$, if

$$
\zeta(x, 0)=\text { identity }(x \in V), \zeta_{t}(x, t)=t
$$

and if $\zeta_{x}(x, t)$ is local automorphism of $M$ for each fixed $t$ where

$$
\zeta(x, t)=\left(\zeta_{x}(x, t), \zeta_{t}(x, t)\right), \quad x \in V, t \in(-\varepsilon, \varepsilon) .
$$

For each open set $V$ of $M$, we set

$$
A(V)=\text { ggerm of } \zeta \text { on } V \times 0\} .
$$

which is a group. By the restriction $A(V) \rightarrow A\left(V^{\prime}\right)$ for $V \supset V^{\prime}$, $\{A(V)\}$ is a presheaf of group over $M$ and induces a sheaf $[A]$ over $M$.

Definition. The sheaf $[A]$ is the sheaf of germs of local automorphisms depending differentiably on 1-parameter for $(B, \Gamma)$ structure $s_{0}$.

Lemma 6. For each open set $V$ of $M$ where $V$ has a regular map $\psi$ of $s_{0}$, there exists an onto-map $\pi: \Pi(V) \rightarrow A(V)$.

Proof. For $\bar{\psi}=(\psi$, germ of $\gamma$ on $\psi(V)) \in \Pi(V),(\gamma \in \Gamma \times I)$, if we set $\tilde{\psi}(x, t)=(\psi(x), t), \quad(x \in V, t \in I)$ and $\tilde{\gamma}(y, t)=(\gamma(y, 0), t)$, $(y \in \psi(V), t \ni I)$, then $\tilde{\psi} \in B \times I(V \times I), \tilde{\gamma} \in \Gamma \times I$ and $(\tilde{\gamma})^{-1} \cdot \gamma \in \Gamma \times I$. Hence, there exists an open set $W$ of $M \times I$ such that $W \cap(M \times 0)=V$, $\tilde{\psi}(W)<$ (domain of $\gamma$ ) and $\tilde{\psi}^{-1} \cdot \tilde{\gamma}^{-1} \cdot \gamma \cdot \widetilde{\psi}$ can be defined on $W$. Since $\tilde{\gamma}^{-1} \cdot \gamma \mid \psi(V)=$ identity and since $\tilde{\psi}^{-1} \cdot \tilde{\gamma}^{-1} \cdot \gamma \cdot \tilde{\psi}$ is a local automorphism of $V$ depending differentiably 1-parameter for $s_{0}$, we see that (germ 
of $\tilde{\psi}^{-1} \cdot \tilde{\gamma}^{-1} \cdot \gamma \cdot \tilde{\psi}$ on $\left.V\right)$ is an element $\pi \bar{\psi}$ of $A(V)$ and the correspondence $\bar{\psi} \rightarrow \pi \cdot \bar{\psi}$ gives a map $\pi: \Pi(V) \rightarrow A(V)$.

Conversely, let (germ of $\zeta$ on $V$ ) be an element $\bar{\zeta}$ of $A(V)$ where $\zeta$ is a local diffeomorphism of an open set of $M \times I$ including $V$ such that $\zeta$ gives a local automorphism of $V$ depending 1-parameter. For a regular map $\mathcal{P}$ of $s_{0}$ on $V$,

$\left(\mathscr{P}\right.$, germ of $\widetilde{\mathcal{P}} \zeta \widetilde{\mathcal{P}}^{-1}$ on $\left.\varphi(V)\right)$ where $\widetilde{\mathcal{P}}(x, t)=(\mathscr{P}(x), t)$ is an element $\bar{\psi}$ of $A(V)$ such as $\pi \bar{\psi}=\zeta$, that is, $\pi$ is onto.

We define $H^{1}(M,[A])$ from the presheaf $\{A(V)\}$ in the same manner as we did for $H^{1}(M,[I])$, and we have

Proposition 2. The map $\pi$ induces a bijection $\pi^{*}: H^{1}(M,[\mathrm{II}])$ $\rightarrow H^{1}(M,[A])$.

Proof. For an element $\left\{\bar{\psi}_{\alpha \beta}\right\}=\left\{\psi_{\alpha \beta}\right.$, germ of $\gamma_{\alpha \beta}$ on $\left.\left.\psi_{\alpha \beta}\left(V_{\alpha} \cap V_{\beta}\right)\right)\right\} \in \mathcal{C}^{1}\left(\mathfrak{B}\right.$, II) where $\mathcal{B}=\left\{V_{\alpha}\right\}$ and $\gamma_{\alpha \beta} \in I^{\prime} \times I$, we have

$$
\tilde{\gamma}_{\alpha \beta}(y, t)=\widetilde{\psi}_{\alpha} \tilde{\psi}_{\beta}^{-1}(y, t) \text { where } y \in \psi_{\beta}\left(V_{\beta}\right), t \in I
$$

and

$$
\left.\tilde{\psi}_{\alpha \beta}=\tilde{\psi}_{\beta} \mid V_{\alpha} \cap V_{\beta}\right) \times I \text { where } \tilde{\psi}_{\alpha}=\left(\psi_{\alpha x}(x), t\right) \quad\left(x \in V_{\alpha}\right)
$$

because $\bar{\psi}_{\alpha \alpha} \cdot \bar{\psi}_{\alpha \beta}=\bar{\psi}_{\alpha \beta} \cdot \bar{\psi}_{\beta \beta}$. Since

$$
\begin{aligned}
& \left(\tilde{\psi}_{\alpha \beta \beta}^{-1} \cdot\left(\tilde{\gamma}_{\alpha \beta}\right)^{-1} \cdot \gamma_{\alpha \beta} \cdot \tilde{\psi}_{\alpha \beta}\right) \cdot\left(\tilde{\psi}_{\beta \gamma}^{-1} \cdot\left(\tilde{\gamma}_{\beta \gamma}\right)^{-1} \cdot \gamma_{\beta \gamma} \cdot \tilde{\psi}_{\beta \gamma}\right)=\tilde{\psi}_{\alpha}^{-1} \cdot \gamma_{\alpha \beta} \cdot \gamma_{\beta \gamma} \cdot \tilde{\psi}_{\gamma} \\
& =\tilde{\psi}_{\gamma}^{-1} \cdot \tilde{\psi}_{\gamma \gamma} \cdot \tilde{\psi}_{\alpha}^{-1} \cdot \gamma_{\alpha \gamma} \cdot \tilde{\psi}_{\gamma \gamma}=\tilde{\psi}_{\alpha \gamma}^{-1} \cdot\left(\tilde{\gamma}_{\alpha \beta}\right)^{-1} \cdot \gamma_{\alpha \gamma} \cdot \widetilde{\psi}_{\alpha \gamma}
\end{aligned}
$$

then $\left\{\pi \tau_{\alpha \beta}\right\}$ is an element of $\mathcal{C}^{1}\left(\mathfrak{Z}^{3}, A\right)$ and moreover this correspondence $\left\{\bar{\psi}_{\alpha \beta}\right\} \rightarrow\left\{\pi \bar{\psi}_{\alpha \beta}\right\}$ gives a map from $\mathcal{C}^{1}(\mathfrak{B}, \Pi)$ onto $\mathcal{C}^{1}(\mathfrak{B}, A)$ by Lemma 5 . If two elements $\left\{\bar{\psi}_{\alpha ; \beta}^{1}\right\}$ and $\left\{\bar{\psi}_{\alpha ; \beta}^{2}\right\}$ of $\mathcal{C}^{1}(\mathfrak{B}, \Pi)$ are cohomologous, then there exists an element $\bar{\psi}_{\alpha}=\left(\psi_{\alpha \alpha}^{1}\right.$, germ of $\gamma_{\alpha}$ on $\left.\psi_{\alpha \alpha}^{1}\left(V_{\alpha}\right)\right)$ of $\Pi\left(V_{\alpha}\right)$ for each $V_{\alpha}$, such that $\bar{\psi}_{\alpha} \cdot \bar{\psi}_{\alpha \beta}^{1}=\bar{\psi}_{\alpha \beta}^{2} \cdot \bar{\psi}_{\beta}$ and $\gamma_{\alpha} \cdot \gamma_{\alpha \beta}^{1}=\gamma_{\alpha \beta}^{2} \cdot \gamma_{\beta}$ on a suitable domain including $\psi_{\alpha \beta}^{1}\left(V_{\alpha} \cap V_{\beta}\right)$. Then

$$
\begin{aligned}
& \left(\left(\widetilde{\psi}_{\alpha}^{2}\right)^{-1} \gamma_{\alpha} \tilde{\psi}_{\alpha}^{1}\right) \cdot\left(\left(\widetilde{\psi}_{\beta}^{1}\right)^{-1} \cdot\left(\tilde{\gamma}_{\alpha \beta}^{1}\right)^{-1} \cdot \gamma_{\alpha \beta}^{1} \cdot \widetilde{\psi}_{\beta}^{1}\right)=\left(\tilde{\psi}_{\alpha}^{2}\right)^{-1} \cdot \gamma_{\alpha} \cdot \gamma_{\alpha \beta}^{1} \widetilde{\psi}_{\beta}^{1} \\
& =\left(\widetilde{\psi}_{\alpha}^{2}\right)^{-1} \cdot \gamma_{\alpha \beta}^{2} \cdot \gamma_{\beta} \cdot \widetilde{\psi}_{\beta}^{1}=\left(\left(\tilde{\psi}_{\beta}^{2}\right)^{-1} \cdot\left(\tilde{\gamma}_{\alpha \beta}^{2}\right)^{-1} \cdot \gamma_{\alpha \beta}^{2} \cdot \widetilde{\psi}_{\beta}^{2}\right) \cdot\left(\left(\tilde{\psi}_{\beta}^{2}\right)^{-1} \cdot \gamma_{\beta} \cdot \widetilde{\psi}_{\beta}^{1}\right)
\end{aligned}
$$

on a suitable open set of $M \times I$ including $V_{\alpha} \cap V_{\beta}(\neq \Phi)$. Therefore, if we set

$$
\bar{\zeta}_{\infty}=\left(\text { germ of }\left(\widetilde{\psi}_{\omega}^{2}\right)^{-1} \cdot \gamma_{\infty} \cdot \widetilde{\psi}_{\alpha}^{1} \text { on } V_{\infty}\right) \in A\left(V_{\alpha}\right),
$$

we have $\bar{\zeta}_{\alpha}\left(\pi \bar{\psi}_{\alpha \beta}^{1}\right)=\left(\pi \bar{\psi}_{\alpha \beta}^{1}\right) \bar{\zeta}_{\beta}$ on $V_{\alpha} \cap V_{\beta}$, i.e. $\left\{\pi \overline{\gamma_{\alpha \beta}^{1}}\right\},\left\{\pi \bar{\psi}_{\alpha \beta}^{2}\right\}$ are 
cohomologous.

Conversely, if $\left\{\pi \bar{\psi}_{\alpha, \beta}^{1}\right\}$ and $\left\{\pi \bar{\psi}_{\alpha \beta}^{2}\right\}$ are cohomologous in $\mathcal{C}^{1}(\mathfrak{B}, A)$, then there exists, for each $\alpha$, a local diffeomorphism $\zeta_{\alpha}$ on an open set $W_{\alpha}$ of $M \times I$ including $V_{\alpha}$ such that $\bar{\zeta}_{\alpha}\left(\pi \bar{\psi}_{\alpha \beta}^{1}\right)=\left(\pi \bar{\psi}_{\alpha \beta}^{2}\right) \bar{\zeta}_{\beta}$ where $\bar{\zeta}_{\alpha}$ is the germ of $\zeta_{\alpha}$ on $V_{\alpha}$, and such that $\zeta_{\alpha}\left(\left(\tilde{\Psi}^{1}\right)^{-1} \cdot\left(\tilde{\gamma}^{1}\right)^{-1} \cdot \gamma_{\alpha \beta}^{1} \cdot \widetilde{\psi}_{\beta}^{1}\right)$ and $\left(\left(\widetilde{\psi}_{\beta}^{2}\right)^{-1} \cdot\left(\tilde{\gamma}_{\alpha \beta}^{2}\right)^{-1} \cdot \gamma_{\alpha \beta}^{2} \cdot \widetilde{\psi}^{2}\right) \zeta_{\beta}$ can be defined and are equal on $W_{\alpha} \cap W_{\beta}(=\Phi)$. If we set $\gamma_{\alpha}=\tilde{\psi}_{\alpha}^{2} \zeta_{\alpha}\left(\tilde{\psi}_{\alpha}^{1}\right)^{-1}$ on $W_{\alpha}$, then

$$
\gamma_{\alpha} \gamma_{\alpha \beta}^{1}=\gamma_{\alpha} \tilde{\psi}_{\alpha}^{1}\left(\tilde{\psi}_{\beta}^{1}\right)^{-1}\left(\tilde{\gamma}_{\alpha \beta}^{1}\right) \gamma_{\alpha \beta}^{1}=\widetilde{\psi}_{\alpha}^{2}\left(\psi_{\beta}^{2}\right)^{-1}\left(\tilde{\gamma}_{\alpha \beta}^{2}\right)^{-1} \gamma_{\alpha \beta}^{2} \gamma_{\beta}=\gamma_{\alpha \beta}^{2} \gamma_{\beta} .
$$

Therefore, $\left(\psi_{\alpha}\right.$, germ of $\gamma_{\alpha}$ on $\left.\psi_{\alpha}\left(V_{\alpha}\right)\right) \cdot \bar{\psi}_{\alpha \beta}^{1}=\bar{\psi}_{\alpha}^{2} \cdot\left(\psi_{\beta}\right.$, germ of $\gamma_{\beta}$ on $\psi_{\beta}\left(V_{\beta}\left(V_{\beta}\right)\right)$, that is, $\left\{\bar{\psi}_{\alpha \beta}^{1}\right\}$ and $\left\{\bar{\psi}_{\alpha \beta}^{2}\right\}$ are cohomologous in $\mathcal{C}^{1}(\mathfrak{B}, \Pi)$.

From Proposition 1 and Proposition 2, we have

Theorem 1. There exists a bijection $\bar{D}\left(s_{0}\right) \rightarrow H^{1}(M,[A])$.

\section{$\$ 5$. Cross-sections of a differentiable bundle}

Let $F$ be a differentiable manifold and $G$ be an effective differentiable transformation group on $F$ and let $\Gamma_{0}$ be the pseudogroup of all local diffeomorphisms of $R^{n}$. For each element $\gamma_{0}$ of $\Gamma_{0}$ whose domain is $U$, we define a diffeomorphism $\tau\left(\gamma_{0}\right): F \times U \rightarrow$ $F \times \gamma_{0}(U)$ such that $\tau\left(\gamma_{0}\right)(x, f)=\left(\gamma_{0}(x), \tau_{F}(x, f)\right)$ and for each fixed $x, \tau_{F}$ is a transformation of $F$ by $G$. Differentiable cross-sections of $F \times R^{n}$ over $U$ can be transformed to differentiable cross-sections over $\gamma_{0}(U)$ by $\tau\left(\gamma_{0}\right)$. If we denote by $\widetilde{B}$ the space of germs of differentiable cross-sections of $F \times R^{n}$ over $R^{n}$, then $\widetilde{B}$ is a topological space with a differentiable structure and $\tau\left(\gamma_{0}\right)$ induces a local diffeomorphism of $\widetilde{B}$. Then $\Gamma_{0}$ defines a pseudogroup $\tilde{\Gamma}$ of local diffeomorphisms of $\widetilde{B}$ associated to $\tau$. Hence we can consider differentiable $(\widetilde{B}, \tilde{\Gamma})$-structures.

On the other hand, let $\left\{U_{i}, \varphi_{i}\right\}$ be a coordinate system of the differentiable structure of $M$, then $\left\{U_{i}, \mathcal{P}_{i}\right\}, F, G$, and $\tau$ define a differentiable fibre bundle $\mathscr{B}$ with the fibre $F$, the structure group $G$, the base space $M$, the bundle space $X$ and the projection $p$. We say $\mathcal{B}$ an $F$-bundle $\tau$-associated to the differentiable structure of $M$ (or a differentiable $F$-bundle) and $\left\{U_{i}, \mathcal{P}_{i}\right\}$ a coordinate system of $\mathcal{B}$. The diffeomorphism $\mathcal{\rho}_{i}: U_{i} \rightarrow R^{n}$ induces a fibre-preserving diffeomorphism $p_{i}^{*}: p^{-1}\left(U_{i}\right) \rightarrow \mathcal{P}_{i}\left(U_{i}\right) \times F$ and 


$$
\mathcal{P}_{j}^{*}\left(\mathcal{P}_{i}^{*}\right)^{-1} \mid \mathcal{P}_{i}\left(U_{i} \cap U_{j}\right) \times F=\tau\left(\mathcal{P}_{j} \mathcal{P}_{i}^{-1} \mid \mathcal{P}_{i}\left(U_{i} \cap U_{j}\right)\right) \quad \text { for } \quad U_{i} \cap U_{j} \neq \Phi .
$$

If $c$ is a differentiable cross-section of $\mathcal{B}$ over $M$, the map $\varphi_{i}^{*} \cdot c \mid U_{i}$ can be regarded as a diffeomorphism $c_{i}$ of $U_{i}$ into $\widetilde{B}$ and $c_{j} \cdot c_{i}^{-1} \mid c_{i}\left(U_{i} \cap U_{j}\right) \in \tilde{\Gamma}$, then $\left\{U_{i}, c_{i}\right\}$ is a coordinate system of a differentiable $(\widetilde{B}, \tilde{\Gamma})$-structure $s$ and $s$ is independent of the coordinate system $\left\{\mathcal{P}_{i}, U_{i}\right\}$ of $\mathcal{B}$. Therefor we have a map $C:\{c\} \rightarrow H^{\circ}\left(M,[\tilde{B} / \tilde{\Gamma}]_{M}\right)$ where $\{c\}$ is the set of all differentiable cross-sections of $\mathcal{B}$ over $M$.

Lemma 7. The map $C$ is a bijection.

Proof. We can take a coordinate system $\left\{U_{i}, \overline{\mathcal{P}}_{i}\right\}$ for $s \in H^{0}\left(M,[\tilde{B} / \tilde{\Gamma}]_{M}\right)$ such that $\left\{U_{i}, \mathscr{P}_{i}\right\}$ is a coordinate system of $\mathscr{B}$ where $\varphi_{i}=p_{0} \cdot \overline{\mathcal{P}}_{i}, p_{0}$ is the projection of sheaf $\widetilde{B} \rightarrow R^{n}$ and $\mathcal{P}_{i}^{*}: p^{-1}\left(U_{i}\right) \rightarrow \mathcal{P}_{i}\left(U_{i}\right) \times F$ is a coordinate function induced from $\mathcal{P}_{i}$. Then $\left(\mathcal{P}_{i}^{*}\right)^{-1} \overline{\mathcal{P}}_{i}(U)$ is a cross-section $s_{i}$ over $U_{i}$ for $\mathcal{B}$ and

$$
\left(\mathcal{P}_{j}^{*}\right)\left(\rho_{i}^{*}\right)^{-1}\left|\overline{\mathcal{P}}_{i}\left(U_{i} \cap U_{j}\right)=\tau\left(\rho_{j} \bullet \rho_{i}^{-1}\right)\right| \overline{\mathcal{P}}_{i}\left(U_{i} \cap U_{j}\right)=\overline{\mathcal{P}}_{j} \overline{\mathcal{P}}_{i}^{-1} \mid \overline{\mathcal{P}}_{i}\left(U_{i} \cap U_{j}\right)
$$

for $U_{i} \cap U_{j} \neq \Phi$ and so

$$
\begin{aligned}
& \left.s_{i} \mid U_{i} \cap U_{j}\right)=\left(\mathcal{P}_{i}^{*}\right)^{-1} \overline{\mathcal{P}}_{i} \mid\left(U_{i} \cap U_{j}\right)=\left(\left(_{j}^{*}\right)^{-1} \mathscr{P}_{j}^{*}\left(\mathcal{P}_{i}^{*}\right)^{-1} \overline{\mathcal{P}}_{i} \overline{\mathcal{P}}_{\mathcal{j}}^{-1} \overline{\mathcal{P}}_{j} \mid U_{i} \cap U_{j}\right. \\
& \quad=\left(\mathcal{P}_{j}^{*}\right)^{-1} \overline{\mathcal{P}}_{j}\left|U_{i} \cap U_{j}=s_{j}\right| U_{i} \cap U_{j},
\end{aligned}
$$

hence $\left\{s_{i}\right\}$ is a cross-section $c$ over $M$. The correspondence $s \rightarrow c$ defines a correspondence $S: H^{0}\left(M,[\widetilde{B} / \tilde{\Gamma}]_{M}\right) \rightarrow\{c\}$ and $S \cdot C=$ identity, $C \cdot S=$ identity

Then we have

Theorem 2. Differentiable cross-sections of the differentiable F-bundle are differentiable $(\widetilde{B}, \tilde{\Gamma})$-structures.

Remark. The proof of Lemma 7 ensures that $C$ gives a bijection of the set of differentiable cross-sections over an open set $U$ of $M$ onto the set of sections of $[\widetilde{B} / \tilde{\Gamma}]_{M}$ over $U$.

\section{§6. Deformations of differentiable cross-sections of the differen- tiable bundle}

From the differentiable $F$-bundle $\mathscr{B}(X, M, F, G)$, a differentiable $F$-bundle $\mathscr{B} \times I(X \times I, M \times I, F, G)$ is naturally defined. As for the coordinate system $\left\{U_{i}, \mathcal{P}_{i}\right\}$ of $\mathscr{B} \times I, \mathcal{P}_{i}$ can be taken to be diffeo- 
On deformations of cross-sections of a differentiable fibre bundle 223 morphisms of $U_{i}$ into $R^{n} \times I$ such as $\mathcal{P}_{i, t}(x, t)=t$ where $\mathcal{P}_{i}=\left(\varphi_{i, x}(x, t), \varphi_{i, t}(x, t)\right),(x, t) \in U$. Differentiable cross-sections $\tilde{d}$ of $\mathcal{B} \times I$ define cross-sections $c$ of $\mathscr{B}$ by the restriction on $M \times 0$, and $\tilde{d}$ is called a (differentiable) deformation of $c$.

Definition. A deformation $\tilde{d}$ of a given cross-section $c_{0}$ of $\mathcal{B}$ is locally trivial if there exist an open neighborhood $U$ relative to $M \times I$ for each point of $M$ and a diffeomorphism $\xi$ from $U$ into $M \times I$ such as

$$
\xi_{t}(x, t)=t, \quad \xi(x, 0)=i d e n t i t y \text { and } \tilde{d}=\xi^{*} c_{0},
$$

where $\xi^{*}$ is a local bundle-automorphism induced by $\xi, \widetilde{c}_{0}(x, t)=\left(c_{0}(x), t\right)$ and $\xi(x, t)=\left(\xi_{x}(x, t), \xi_{t}(x, t)\right),((x, t) \in U)$.

Now, we take $\widetilde{B}, \tilde{\Gamma}$ as $B, \Gamma$ in $\S \S 2-3$, then $\widetilde{B} \times I, \tilde{\Gamma} \times I$, $[\widetilde{B} \times I / \tilde{\Gamma} \times I]_{M \times I}, D\left(\tilde{s}_{0}\right)\left(\tilde{s}_{0} \in H^{0}\left(M,[\tilde{B} / \tilde{\Gamma}]_{M}\right)\right),[\tilde{\Pi}], \tilde{D}\left(s_{0}\right)$ and $[\tilde{A}]$ take the place of $B \times I, \Gamma \times I,[B \times I / \Gamma \times I]_{M \times I}, D\left(s_{0}\right),[\Pi], \bar{D}\left(s_{0}\right)$ and $[A]$, respectively. If we apply Theorem 1 to this case, we have

Proposition 3. We have a bijection $\widetilde{D}\left(s_{0}\right) \rightarrow H^{1}(M,[A])$.

Let $\overparen{B \times I}$ be the space of germs of differentiable cross-sections of the product bundle $F \times\left(R^{n} \times I\right)$ over $R^{n} \times I$ and let $\widetilde{\Gamma \times I}$ be the pseudogroup of local diffeomorphisms of $\overparen{B \times I}$ induced by local diffeomorphisms of $R^{n} \times I$ as in $\S 5$. Then

Lemma 8. $H^{0}\left(M \times I, \quad[\widetilde{B} \times I / \tilde{\Gamma} \times I]_{M \times I}\right)$ is a sub-set of $\left.H^{0}(M \times I, \widetilde{[B \times I} / \widetilde{\Gamma \times I}]_{M \times I}\right)$.

Proof. $\widetilde{B} \times I$ is a sub-space of $\widetilde{B \times I}$ and $\tilde{\Gamma} \times I$ is a sub-pseudoproup of $\overparen{\Gamma \times I}$. The set $\widetilde{B} \times I(U)$ is a sub-set of $\widetilde{B \times I}(U)$ for each open set $U$ of $M \times I$. If

$$
\mathcal{P}, \psi \in \widetilde{B} \times I(U) \text { and } \boldsymbol{\rho} \cdot \psi^{-1}=\gamma \in \widetilde{\Gamma \times I},
$$

then $\gamma \in \tilde{\Gamma} \times I$ and therefore $\widetilde{B} \times I / \tilde{\Gamma} \times I(U) \subset \widetilde{B \times I} / \widetilde{\Gamma \times I}(U)$ by Lemma 1. Therefore, $[\widetilde{B} \times I / \tilde{\Gamma} \times I]_{M \times I} \subset[\widetilde{B \times I} / \widetilde{\Gamma \times I}]_{M \times I}$ since $r_{U^{\prime}}^{U}(\widetilde{B} \times I(U)) \subset \widetilde{B} \times I\left(U^{\prime}\right)$ for $U \supset U^{\prime}$.

If we apply Lemma 7 to the set $\{\tilde{c}\}$ of differentiable crosssections of $\mathscr{B} \times I$ and $\left.H^{\circ}(M \times I, \widetilde{[B \times I} / \widetilde{\Gamma \times I}]_{M \times I}\right)$, we have a bijection 


$$
\{\tilde{c}\} \underset{\widetilde{S}}{\tilde{C}} H^{0}\left(M \times I,[\widetilde{B \times I} / \widetilde{\Gamma \times I}]_{M \times I}\right) .
$$

Definition. Locally trivial deformations $\widetilde{d^{1}}$ and $\widetilde{d^{2}}$ of $c_{0}$ are locally equivalent if there exist a positive number number $\varepsilon<1$ and a diffeomorphism $\xi$ from $M \times(-\varepsilon, \varepsilon)$ into $M \times I$ such that

1. $\xi_{t}(x, t)$ is independent of $x$ for $(x, t) \in M \times(-\varepsilon, \varepsilon)$,

2. $\xi(x, 0)=$ identity,

3. $\widetilde{d}^{1} \mid \xi(x, t)=\xi^{*}\left(\widetilde{d}^{2}(x, t)\right)$,

where $\xi(x, t)=\left(\xi_{x}(x, t), \xi_{t}(x, t)\right)$ and $\xi^{*}$ is a bundle map induced by $\xi$.

If we set $\tilde{s}_{0}=C\left(c_{0}\right)$ where $c_{0}$ is a given cross-section of $\mathcal{B}$, then $\tilde{S}$ maps bijectively $D\left(\tilde{s}_{0}\right)$ onto a sub-set $E\left(c_{0}\right)$ of the set of locally trivial deformations of $c_{0}$.

Lemma 9. For each locally trivial deformation $\tilde{d}$ of $c_{0}$, there exists an element $\tilde{d}^{\prime}$ of $E\left(c_{0}\right)$ such that $\tilde{d}$ and $\tilde{d}^{\prime}$ are locally equivalent.

Proof. Let $\left\{U_{j}, \mathscr{P}_{j}, j \in J\right\}$ be a coordinate system of $\mathscr{B}$. Since $\widetilde{d}$ is a locally trivial deformation of $c_{0}$ and since $M$ is compact, there are a finite covering $\left\{U_{k}^{\prime}, k \in K\right\}$ of $M \times 0$ by open sets of $M \times I$ and diffeomorphisms $\xi_{k}$ of $U_{k}$ into $M \times I$ for each $k \in K$, such that the covering $\left\{U_{k}^{\prime}\right\}$ is a refinement of the covering $\left\{U_{j} ; U_{j} \cap(M \times 0) \neq \Phi, j \in J^{\prime}\right\}$ of $M \times 0$ (with the index injection of the refinement $\left.\kappa: K \rightarrow J^{\prime}\right), \xi_{k}^{*} \tau_{0}=\widetilde{d}$ on $U_{k}^{\prime}$ and $\xi_{k}\left(U_{k}^{\prime}\right) \subset U_{k}$. Then

$$
\begin{aligned}
& \mathcal{P}_{\kappa^{\prime}(k)}^{*} \tilde{d}(x, t)=\mathcal{P}_{\kappa^{(}(k)}^{*}\left(\xi_{k} \widetilde{c}_{0}\right)(x, t)=\mathcal{P}_{\kappa^{\prime}(k)}^{*} \widetilde{c}_{0}\left(\xi_{k}(x, t)\right)=\mathcal{P}_{\kappa^{\prime}(k)}^{*} \widetilde{c}_{0}\left(\xi_{k, x}(x, t), t\right) \\
& \left.\left.==\mathcal{P}_{\kappa^{\prime}(k)}^{*} \widetilde{c}_{0}\left(\xi_{k, x}(x, t)\right), t\right)\right) \subset\left(\tilde{s}_{\kappa(k)}\left(\xi_{k, x}(x, t)\right)\right) \times I \subset \widetilde{B} \times I
\end{aligned}
$$

where $(x, t) \in U^{\prime}$ and $\xi_{k}(x, t)=\left(\xi_{k, x}(x, t), t\right)$, hence $\widetilde{C}\left(\widetilde{d} \mid U_{k}^{\prime}\right)$ is a section of $[\tilde{B} \times I / \widetilde{\Gamma} \times I]_{M \times I}$ over $U_{k}^{\prime}$ by Lemark in $\S 5$. If we take a positive number $\varepsilon$ such as $M \times(-\varepsilon, \varepsilon) \subset \bigcup_{k \in K} U_{k}^{\prime}$, then $\tilde{C}(\tilde{d} \mid M \times(-\varepsilon, \varepsilon))$ is a section of $[\tilde{B} \times I / \tilde{\Gamma} \times I]_{M \times I}$ over $M \times(-\varepsilon, \varepsilon)$. By Lemma 4, this section can be extended over $M \times I$ which is an element $d$ of $D\left(s_{0}\right)$. Then $\widetilde{S} \cdot d \in E\left(c_{0}\right)$ and

$$
\widetilde{S} \cdot d|M \times(-\varepsilon, \varepsilon)=\widetilde{S} \cdot \widetilde{C}(\widetilde{d} \mid M \times(-\varepsilon, \varepsilon))=\widetilde{d}| M \times(-\varepsilon, \varepsilon)
$$

i.e. $\widetilde{S} \cdot d$ and $\tilde{d}$ are equal on $M \times(-\varepsilon, \varepsilon)$. 
On deformations of cross-sections of a differentiable fibre bundle 225

By definitions, the local equivalence of locally trivial deformations of $c_{0}$ applied to $E\left(c_{0}\right)$ and local equivalence of $D\left(\tilde{s}_{0}\right)$ are compatible with the bijection $E\left(c_{0}\right) \rightarrow D\left(\tilde{s}_{0}\right)$. Then, by Lemma 9 we have

Proposition 4. The set of local equivalence clases of all locally trivial deformations of $c_{0}$ can be identified with the set $\widetilde{D}\left(\tilde{s}_{0}\right)$ of local equivalence classes of $D\left(\tilde{s}_{0}\right)$.

A local diffeomorphism $\xi_{V}$ of an open set $V$ of $M$ into $M$ is said to be a local automorphism of $V$ for the cross-section $c_{0}$, if $c_{0}\left|\xi_{V}(x)=\xi_{V}^{*} \cdot c\right| x$ where $\xi_{V}^{*}$ is a local bundle map induced by $\xi_{V}$.

Definition. A local diffeomorphism $\zeta$ of an open set $V \times(-\varepsilon, \varepsilon)$ of $M \times I$ into $M \times I$ is a local automorphism on $V$ depending differentiably on 1-parameter for the cross-section $c_{0}$ if $\zeta_{t}(x, t)=t$, and for each fixed $t, \zeta_{x}(x, t)$ is a local automorphism of $V$ for $c_{0}$, where $\zeta(x, t)=\left(\zeta_{x}(x, t), \zeta_{t}(x, t)\right)$

From the definition of the map $C(\S 5$.$) , local automorphisms$ on $V$ depending differentiably on 1-parameter for $c_{0}$ are local automorohisms on $V$ depending differentiably on 1-parameter for the $(\widetilde{B}, \tilde{\Gamma})$-structure $\tilde{s}_{0}=C\left(c_{0}\right)$. Then, the sheaf [M] of germs of local automorphisms depending differentiably for the given crosssection $c_{0}$ of $\mathcal{B}$ is isomorphic to the sheaf [A] for $C\left(c_{0}\right)$.

Therefore, from Proposition 3 and Proposition 4, we have

Theorem 3. There is a one-to-one correspondence between the set of local equivalence classes of locally trivial deformations of the cross-section $c_{0}$ of $\mathcal{B}$ and the cohomology set $H^{1}(M,[M])$.

\section{§7. Remarks}

1. The fibre bundle of positive definite symmetric tensors of the differentiable manifold $M$ is a fibre bundle associated to the differentiable structure of $M$ and its cross-sections are Riemannian metrices on $M$. In this case, our sheaf $[\mathfrak{M}]$ is the sheaf of germs of motions depending differentiably on 1-parameter for the given Riemannian metric $g_{0}$.

2. Though we have discussed " 1 -parameter" to simplify the 
exposition, our theory is valid for " $m$-parameter" by taking $I^{m}$ as the parameter space.

\section{REFERENCES}

[1] Chern, S. S. Pseudo-groupes continus infinis, Colloques de géométrie différentielle, Strasbourg, C.N.R.S. (1953) p.p. 119-136.

[2] Dedecker, P. Introduction aux structures locales, Colloques de géométrie différentielle globale, Bruxelles, C.B.R.M. (1958) p.p. 103-135.

[3] Ehresmann, C. Structures locales et structures infinitésimales, Comptes Rendus, 234 (1952) p.p. 587-589.

[4] - Introduction à la théorie des structures infinitésimales et des pseudogroupes de Lie, Colloque de géométrie différentielle, Strasbourg, C.N.R.S. (1953), p.p. 97-110.

[5] —- Catégories topologique et catégories différentiables, Colloque de géométrie différentielle globale, Bruxelles, C.B.R.M. (1958) p.p. 137-150.

[6] Gray, J. W. Abstract theory of preudo-grougs. Reports seminor in topology, part II. (X), Univ. of Chicago. (1957).

[7] - Some global properties of contact structures. Ann. of Math., 69 (1959), p.p. $421-329$.

[8] Haefliger, A. Structures feuilletées et cohomologie à valeur dans un faiceau de groupoïdes. Comment. Math. Helv., 32 (1957-1958), p.p. 248-329.

[9] Spencer, D. C. Some remarks on perturbation of structure. "Analytic function" Princeton Univ. press, (1960).

[10] _..., Homological analysis and structures. Differential Geometry, Proceedings of Symposia in pure mathematics, III (1961) p.p. 56-86. 